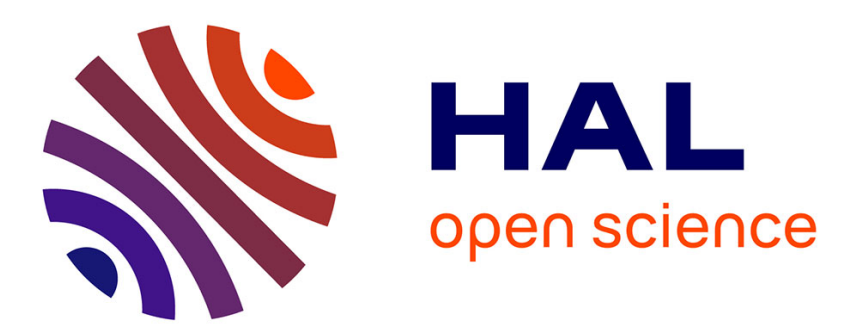

\title{
Cavity growth and rupture of $\beta$-treated zirconium: A crystallographic model
}

Jérôme Crépin, Thierry Bretheau, Daniel Caldemaison

\section{To cite this version:}

Jérôme Crépin, Thierry Bretheau, Daniel Caldemaison. Cavity growth and rupture of $\beta$-treated zirconium: A crystallographic model. Acta Materialia, 1996, 44, pp.4927-4935. 10.1016/S13596454(96)00093-6 . hal-00111518

\section{HAL Id: hal-00111518 https://hal.science/hal-00111518}

Submitted on 18 Mar 2021

HAL is a multi-disciplinary open access archive for the deposit and dissemination of scientific research documents, whether they are published or not. The documents may come from teaching and research institutions in France or abroad, or from public or private research centers.
L'archive ouverte pluridisciplinaire HAL, est destinée au dépôt et à la diffusion de documents scientifiques de niveau recherche, publiés ou non, émanant des établissements d'enseignement et de recherche français ou étrangers, des laboratoires publics ou privés. 


\title{
CAVITY GROWTH AND RUPTURE OF $\beta$-TREATED ZIRCONIUM: A CRYSTALLOGRAPHIC MODEL
}

\author{
J. CRÉPIN, T. BRETHEAU and D. CALDEMAISON \\ Laboratoire de Mécanique des Solides, C.N.R.S. U.R.A. 317, École Polytechnique, \\ 91128 Palaiseau Cedex, France
}

\begin{abstract}
This study aims to understand the damage mechanisms observed in $\beta$-treated zirconium. Damage voids are characterized by a tubular morphology with hexagonal cross-section; their growth kinetics is determined experimentally. From these observations, a model based on the principle of creation of free surface by crystallographic slip permits one to explain the stability of the hexagonal shape and to predict a growth rate closer to the experimental value than traditional models. This improvement is due to the sensitivity of the free surface creation mechanism to the stress concentration factor $k$, which can not be accounted for in models based on continuum mechanics.
\end{abstract}

\begin{abstract}
Résumé-Cette étude a pour but de comprendre les mécanismes d'endommagement observés dans le zirconium traité $\beta$. L'endommagement est caractérisé par des cavités de forme tubulaire à section hexagonale; leur cinétique de croissance est déterminée expérimentalement. A partir de ces observations, un modèle basé sur le principe de création de surface libre par glissement cristallographique permet d'expliquer la stabilité de la forme hexagonale et de prévoir une vitesse de croissance plus proche de la valeur expérimentale que celles prévues par les modèles traditionnels. L'amélioration de la prédiction est due à la sensibilité des mécanismes de création de surface libre vis à vis du facteur de concentration de contrainte $k$, ce qui ne peut être pris en compte dans un modèle basé sur la mécanique des milieux continus.
\end{abstract}

\section{INTRODUCTION}

Zirconium, a material mainly used in the nuclear industry, is characterized by the existence of two allotropic varieties, the $\beta$ phase and the $\alpha$ phase which are b.c.c. and h.c.p. crystals, respectively. The former is found above, and the later below $1138 \mathrm{~K}$. The grade 702 zirconium tested had undergone an $\alpha \rightarrow \beta \rightarrow \alpha$ cycle at a slow cooling rate $\left(\sim 15^{\circ} / \mathrm{s}\right)$ inducing a bainitic transformation. The resulting microstructure consists of colonies of parallel laths with the same crystallographic orientation; the "lath boundaries" are planes where the precipitates of betagenic elements $(\mathrm{Fe}, \mathrm{Cr}, \mathrm{Ni})$ locate. Three deformation mechanisms are activated, prismatic slip $(10 \overline{1} 0)\langle 1 \overline{2} 10\rangle,(10 \overline{1} 2)\langle 10 \overline{11}\rangle$ twinning and $(11 \overline{2} 1)\langle 11 \overline{26}\rangle$ twinning, depending on the colony crystallographic orientation with respect to the tensile axis in accordance with Schmid's law. Prismatic slip is either homogeneous or concentrated in coarse slip lines along the lath boundaries [1]. This last mechanism is the most frequent one.

\section{CHARACTERIZATION OF DAMAGE AND RUPTURE}

\section{Experimental procedure}

Flat tensile test specimens $(20 \times 5 \times 2 \mathrm{~mm})$ were polished mechanically and electrolytically $\left(U=22 \mathrm{~V}, T=300 \mathrm{~K}, t=30 \mathrm{~s}\right.$, in $\mathrm{CH}_{3} \mathrm{COOH}: 80 \%$,
$\mathrm{HCLO}_{4}: 20 \%$ ). Then they were chemically etched (HF:22\%, $\mathrm{HNO}_{3}: 11 \%$, glycerol:67\%, T= $300 \mathrm{~K}$, $t \simeq 5 \mathrm{~s}$ ) to reveal the microstructure.

To follow on the sample surface the deformation mechanisms and the growth rate of damage, a Raith tensile stage located inside a SEM was used with a strain rate of the order of $10^{-4} \mathrm{~s}^{-1}$. Fiducial $1 \mathrm{~mm}^{2}$ gold grids with $5 \mu \mathrm{m}$ pitch deposited on the surface of the sample permit one to follow qualitatively and quantitatively the local deformation mechanisms [2].

The local crystallographic orientation of crystal domains a few $\mu \mathrm{m}^{3}$ large is determined with Electron Backscattering Diffraction (EBSD). This technique, which involves indexing the Kikuchi lines, provides the orientation of the studied crystal relative to a macroscopic reference axis attached to the sample tensile axis. Consequently, with the approximation of uniaxial stress, the Schmid factor of any deformation mechanism can be calculated in each grain.

\section{The sample surfaces during a tensile test}

At the surface of tensile test samples, damage cavities are observed for a global strain between 3 and $6 \%$, far before any necking. Ductile rupture occurs at a global strain of about $10 \%$, necking remaining weak in any case. The first damage cavities always appear in colonies subjected to prismatic slip either concentrated or homogeneous, but never in colonies 


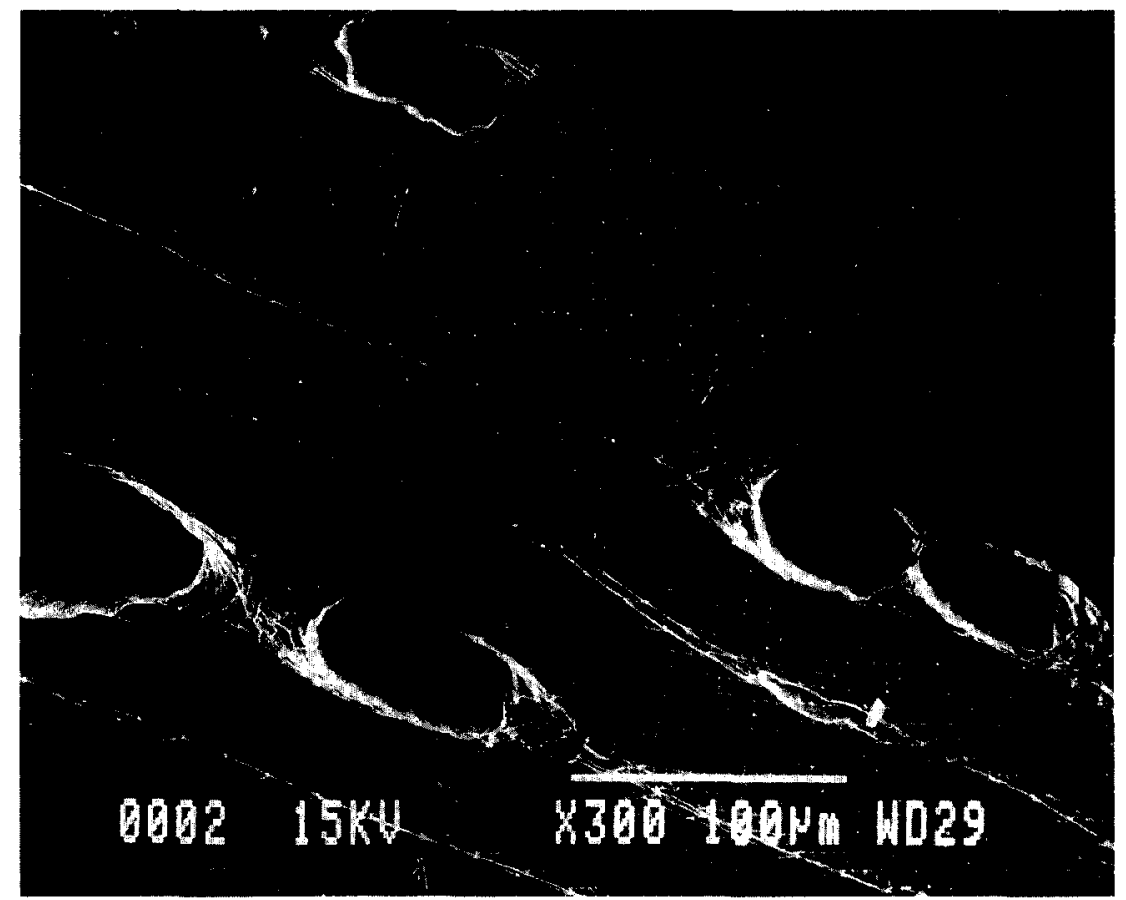

Fig. 1. Cavity alignments parallel to the lath boundaries; localization of deformation along the boundary observed thanks to the deformation of fiducial grids.

subjected to twinning; they appear on the sample surface between the precipitates located at the lath boundaries or inside the lath between the last coarse slip line and the remaining undeformed part of the lath. In a colony, the cavities form alignments parallel to the lath boundaries (Fig. 1). During this growth, the cavities remain contained within one lath and their ultimate size is of the order of the thickness of the lath which is $10 \mu \mathrm{m}$. Cavities can show a peculiar hexagonal shape. Cavity coalescence occurs inside the alignments (Fig. 2) with the possibility of rupture of the ligament between two neighboring alignments. The absence of deformation exhibited by fiducial grids for the laths which are not affected by voids shows that damage remains confined along the lath boundary (Fig. 1).

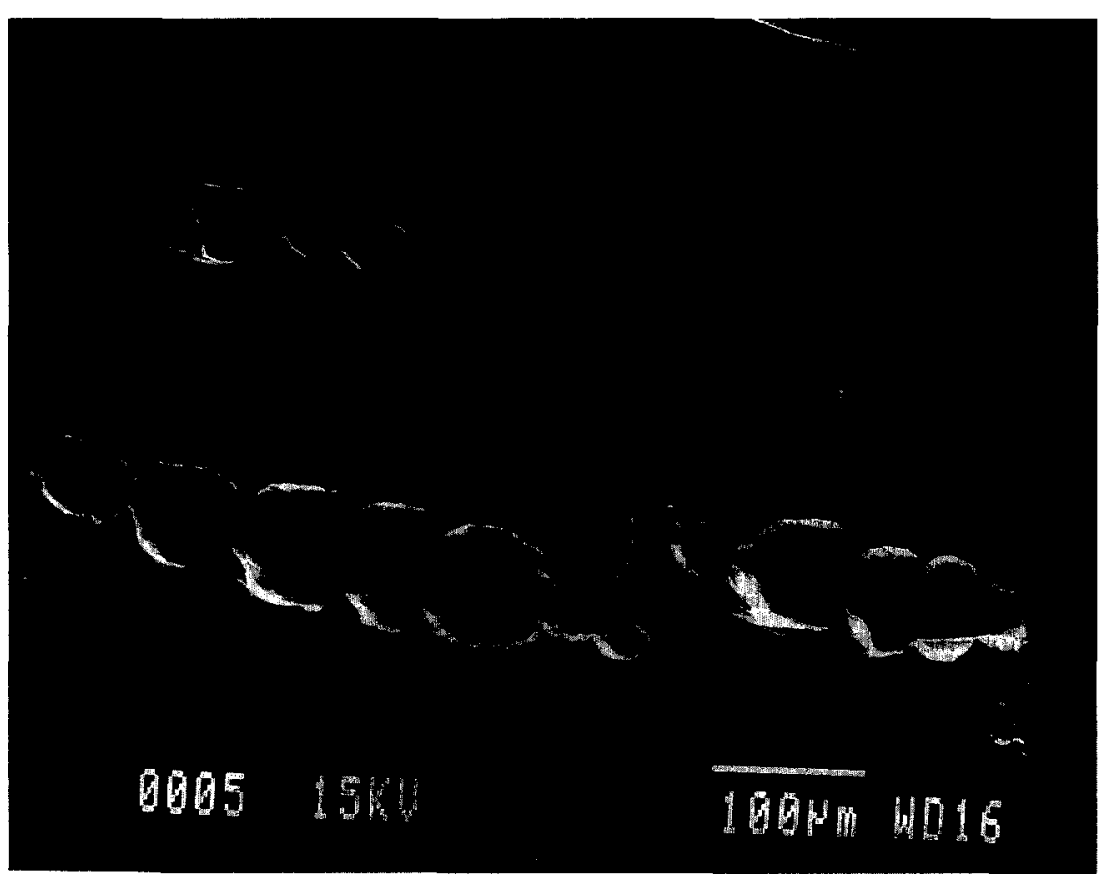

Fig. 2. Cavity coalescence inside alignments; rupture of the ligament between two neighboring alignments. 


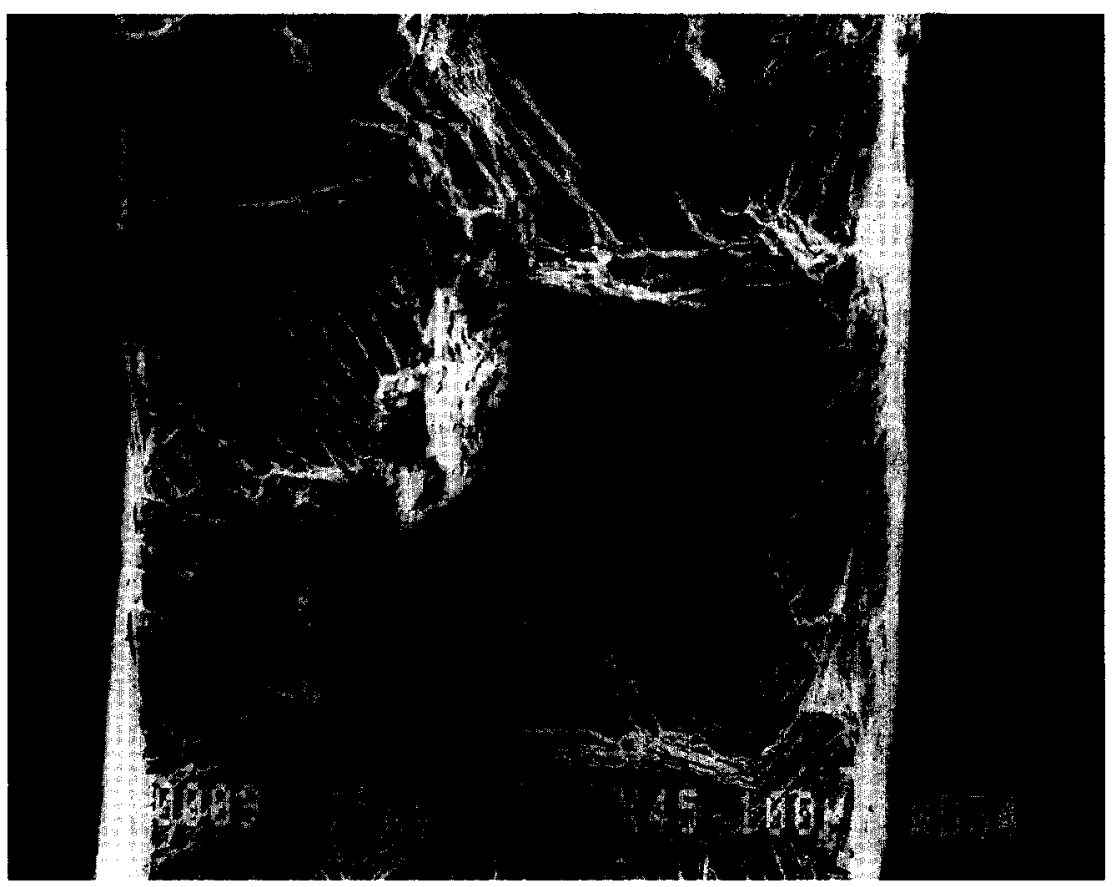

Fig. 3. Observation of the rupture surface.

Another type of damage has been observed at the inter-colony boundaries; it is due to the strong strain incompatibility between colonies which stemmed from a same former $\beta$ grain and have a misorientation of either 60 or $90^{\circ}$ (this misorientation is a consequence of the allotropic transformation). This damage development involves the activation of prismatic slip or twinning according to the orientation of the grain with respect to the tensile axis [1]. Rupture occurs along the cavity alignments. The connection of the cracks developed inside adjacent colonies proceeds either by rupture of the damaged intercolony segregation zones or of the lath extremities.

\section{The rupture surface}

In Fig. 3 the rupture does not seem to be ductile because small circular dimples on the rupture surface are absent. It shows large areas of parallel elongated shapes with a long axis of several hundred micrometers and a small axis of about $10 \mu \mathrm{m}$ corresponding to the size of the colonies. The comparison of the two associated rupture surfaces allows one to conclude that these elongated shapes are large elongated dimples. Such dimples called "fluting zones" were described by Aitchinson and Cox [3] and Spurrier and Scully [4] in the case of stress--corrosion rupture. They reported that the long axis of these dimples is parallel to the local crystalline $\langle c\rangle$ axis. Some zones constituted of small rounded dimples are also detected in the rupture surface, but found to be less numerous than the elongated ones. EDS analysis shows that the zones with small rounded dimples are rich in intermetallic precipitates, whereas zones with elongated large dimples do not have such precipitates.

\section{Relation between the two observed surfaces}

The necessary coherence between the different observations, the sample surfaces and the rupture surface, allows one to characterize the cavity morphology. If the same cavity is observed either on the sample surface [Fig. 4(a)] or on the rupture surface [Fig. 4(b)] it shows, without ambiguity, that it is tubular; an EBSD analysis of the lath orientation permits one to conclude that the tube axis is parallel to the $\langle c\rangle$ crystalline axis. Some of the cavities have a hexagonal cross section with faces parallel to prismatic planes. This probably results from a predominant role of prismatic glide. The parallel ridges in the fracture surface correspond to the remaining ligaments due to the coalescence of these tubular cavities aligned along a lath boundary. The small dimple zones are associated with the rupture of lath extremities necessary to allow the principle crack to propagate from a colony to the neighboring one; the stress state in these zones is triaxial and activates secondary slip systems resulting in the small rounded dimples.

\section{Cavity nucleation}

Cavities can appear either on the precipitates constituting the lath boundaries or inside the lath. Does this mean that two different types of nucleation sites have to be distinguished? Successive polishing of the surface presenting initially a cavity inside a lath, allows one to locate the tubular cavity in the bulk of the sample. It appears that the nucleation had 
occurred at the lath boundary. Thus, it seems that cavities systematically nucleate on the rows of precipitates constituting the lath boundaries; then the location of a cavity appearing inside a lath in the sample surface is due to the angular difference between the tube axis of the cavity, which corresponds to the $\langle c\rangle$ axis of the lath and the plane of the boundary, which is close to a prismatic plane due to the allotropic transformation [1].

\section{THE HEXAGONAL CAVITY}

The main conclusions so far consist of the link between the activation of prismatic slip, principally localized in coarse slip bands, and the occurrence of damage. The main damaging effect is due to tubular cavities, which nucleate on precipitates and have an axis parallel to the local $\langle c\rangle$ axis. The propagation of the cracks is a consequence of the coalescence of these cavities.

In the following, special attention will be paid to the hexagonal cavities (Fig. 5), from the geometrical, mechanical and kinematical point of view.

\section{Experimental characterization}

One particular cavity (Fig. 5) and the slip lines surrounding it have been characterized by means of EBSD technique. The determination of the orien-

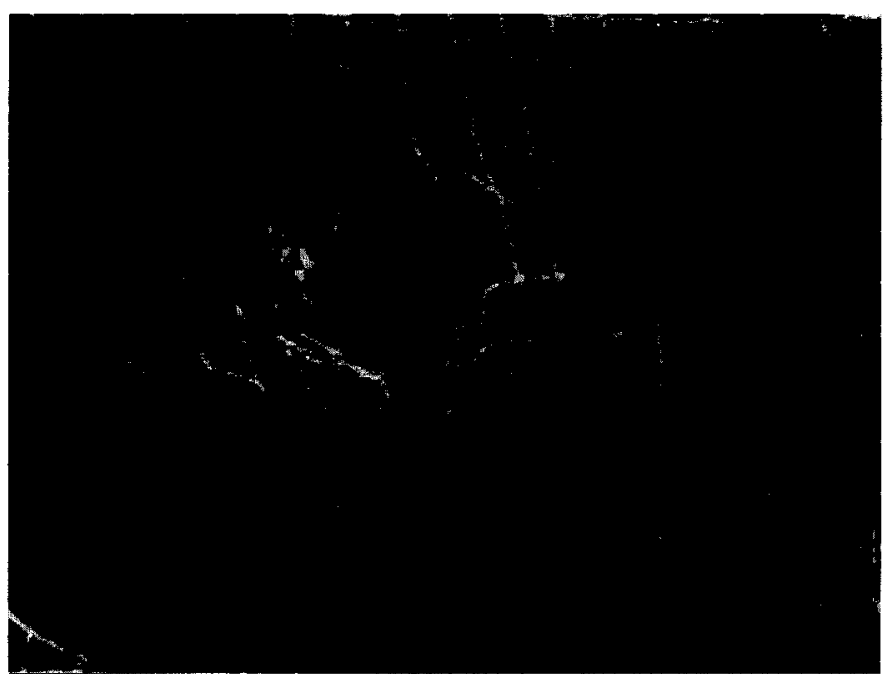

(a)

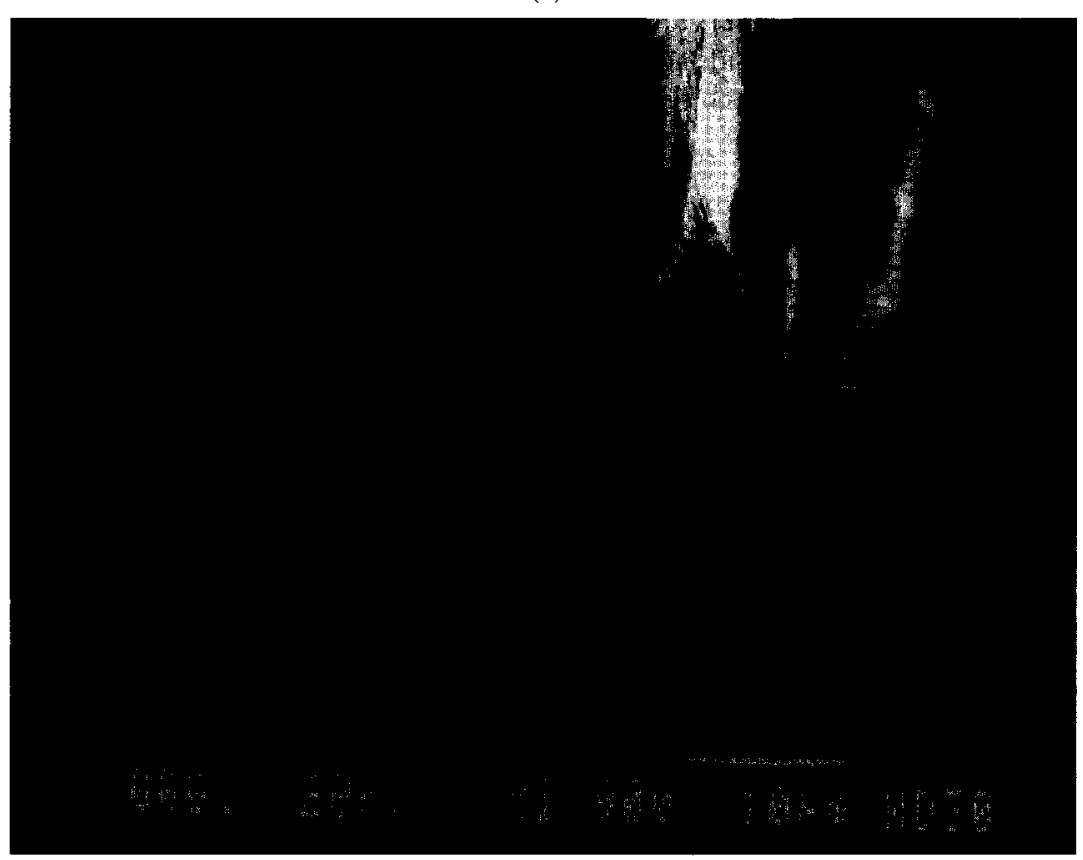

(b)

Fig. 4. (a) Hexagonal cross-section of the cavity observed in the sample surface. (b) Tubular dimple due to the cavity observed on Fig. 4(a) after rupture. 


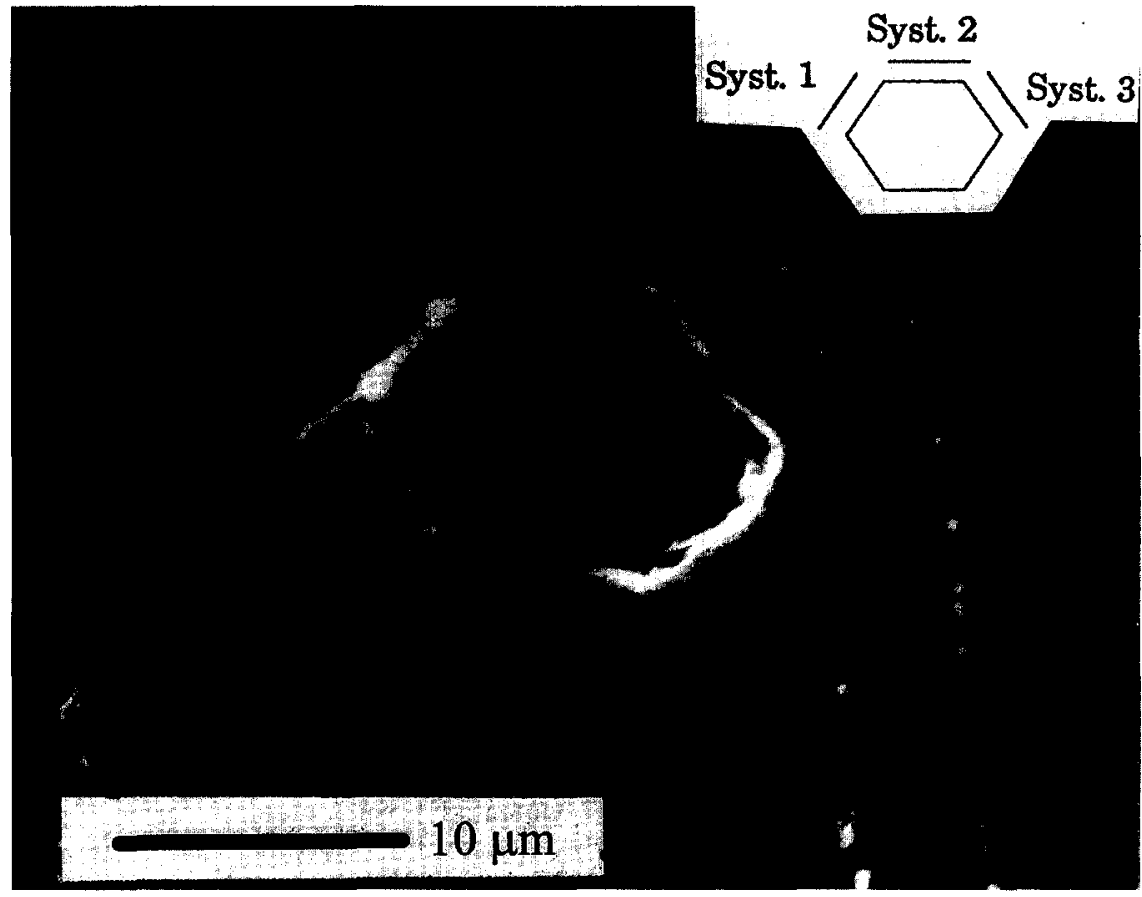

Fig. 5. Hexagonal cavity with the three prismatic slip systems $(1,2,3)$ activated two by two (vertical tensile axis).

tation of the lath in the sample coordinate system permits one to conclude that the local $\langle c\rangle$ axis is quasi-perpendicular to the sample surface $\left(87^{\circ}\right)$, which contains the tensile axis and that the faces of the hexagonal cavity are the three prismatic planes. The slip traces, observed around the cavity, are due to the same prismatic planes. It must be noted that the slip traces can be observed because of the imperfect orthogonality of the $\langle c\rangle$ axis with respect to the sample surface. If the $\langle c\rangle$ axis was orthogonal to the sample surface, the gliding $\langle a\rangle$ dislocations should be parallel to the sample surface and consequently should not leave any trace. The slip systems are activated two by two depending on the considered hexagon angle (thus, in a clockwise rotation one can observe the activation of systems 3 and 2, then 3 and 1, then 1 and 2). The Schmid factor of each prismatic slip system has been determined:

$$
F_{1}=0.49, \quad F_{2}=0.16, \quad F_{3}=0.33 ;
$$

using a hypothesis of uniform stress.

For a distance greater than two or three times the radius of the cavity, but within the same colony, only Systems 1 and 3 are activated (not observable on Fig. 5), the lowest Schmid factor being 0.33 for System 3. The fact that System 2 is solely observed within a distance to the cavity of the order of the radius, although its Schmid factor is weak $\left(F_{2}=0.16\right)$, suggests that the cavity produces a perturbation of the local stress field.

Moreover a video depicting the nucleation and growth of a cavity was recorded during an in situ tensile test (nominal strain rate $\dot{\epsilon}_{x}$ of the order $\left.10^{-4} \mathrm{~s}^{-1}\right)$. This video shows that the cavity appears with a rounded shape $(0.2 \mu \mathrm{m})$ and after few seconds ( $2 \mathrm{~s})$ takes on an hexagonal shape that remains stable during the deformation with a homothetical growth. The cavity growth rate can be written as:

$$
\frac{\dot{R}}{R}=\zeta \dot{\epsilon}_{x} \quad \text { with } \zeta=260
$$

Rice and Tracey [5] studied the case of a spherical cavity of radius $R$ growing in material following a Norton-Hoff law (2):

$$
\sigma=\sigma_{0}\left(\frac{\dot{\epsilon}}{\dot{\epsilon}_{0}}\right)^{m}
$$

they obtained:

$$
\begin{aligned}
& \frac{\dot{R}}{R}=\frac{1}{2}\left(\operatorname{sgn} \sigma_{\mathrm{m}}\right) \dot{\epsilon}_{\mathrm{eq}}\left\{\frac{3}{2} m \frac{\left|\sigma_{\mathrm{m}}\right|}{\sigma_{\mathrm{eq}}}\right. \\
& \left.+(1-m)\left[1+m\left(0,4175+0,144 \chi\left(\operatorname{sgn} \sigma_{\mathrm{m}}\right)\right)\right]\right\}^{1, m}
\end{aligned}
$$

with: $\sigma_{\mathrm{m}}$, hydrostatic stress;

$\sigma_{\mathrm{eq}}$, von Mises equivalent stress;

$\dot{\epsilon}_{e q}$, von Mises equivalent strain rate;

$m$, stress sensitivity factor;

$\chi=+1$ if the axial stress is greater than the radial stress -1 otherwise. 
Also, in the case of uniaxial tension for a Newtonian material $(m=1)$, this expression gives:

$$
\frac{\dot{R}}{R}=\left(\frac{3}{4}\right)\left(\frac{\sigma_{\mathrm{m}}}{\sigma}\right) \dot{\epsilon}_{\mathrm{eq}} .
$$

McClintock [6] studied the case of a cylindrical cavity of radius $R$; for a Newtonian $(m=1)$ material or a perfectly plastic $(m=0)$ material subjected to a uniaxial tension his model predicts a $\zeta$ value of order 1 (Fig. 6). Therefore, the experimental cavity growth rate is much faster than predicted by these models. The experimental observations and contradiction with the prediction pose three questions:

Why are the three prismatic slip systems activated two by two around the cavity? Why is the hexagonal shape stable? Why is the cavity growth rate so high?

\section{Stress field analysis}

To solve the problem analytically, the calculations performed are for an isotropic elastic solid in plane strain. These geometrical restrictions are consistent with the observation of cavity growth by the sole activation of prismatic slip systems. Consider a circular hole of radius $R$ in an infinite plate subjected to a remote uniaxial tensile stress $S$. The stress at any point of the plate in a polar coordinate system $(r, \theta, z)$ is [7]:

$$
\begin{gathered}
\sigma_{\mathrm{r}}=\frac{S}{2}\left(1-\frac{R^{2}}{r^{2}}\right)+\frac{S}{2}\left(1+3 \frac{R^{4}}{r^{4}}-4 \frac{R^{2}}{r^{2}}\right) \cos 2 \theta, \\
\sigma_{\theta}=\frac{S}{2}\left(1+\frac{R^{2}}{r^{2}}\right)-\frac{S}{2}\left(1+3 \frac{R^{4}}{r^{4}}\right) \cos 2 \theta, \\
\sigma_{\mathrm{r} \theta}=-\frac{S}{2}\left(1-3 \frac{R^{4}}{r^{4}}+2 \frac{R^{2}}{r^{2}}\right) \sin 2 \theta .
\end{gathered}
$$

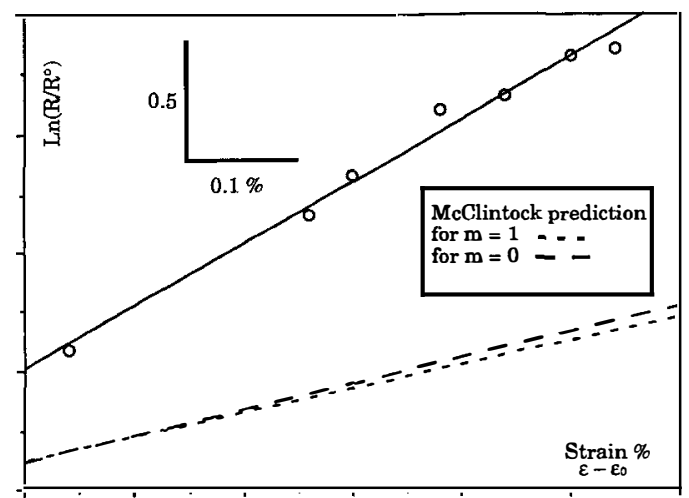

Fig. 6. Comparison between experimental cavity growth rate of zirconium and $\mathrm{McClintock}$ prediction for Newtonian $(m=1)$ and perfectly plastic $(m=0)$ material. $\bigcirc$ Experimental values.

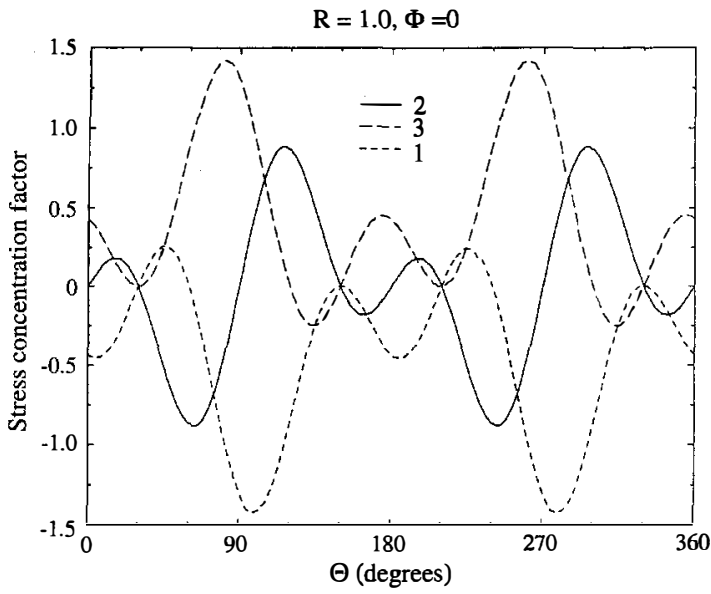

Fig. 7. Evolution of the local stress concentration factor on the three prismatic systems vs $\theta$ around the cavity.

This stress state is now projected on the three potential prismatic slip systems. If the $\langle c\rangle$ axis is considered as the tube axis (in agreement with the obervations) and parallel to the $z$ axis, and if $\phi$ is the rotation angle around this axis between the crystal base and the macroscopic base, it follows that:

$$
\begin{aligned}
& \tau_{1}=\left[\frac{1}{2}(\right.\sqrt{3} \sin 2 \phi-\cos 2 \phi) \sigma_{12} \\
&\left.+\frac{1}{4}(\sin 2 \phi+\sqrt{3} \cos 2 \phi)\left(\sigma_{22}-\sigma_{11}\right)\right] ; \\
& \tau_{2}=\cos 2 \phi \sigma_{12}+\frac{\sin 2 \phi}{2}\left(\sigma_{11}-\sigma_{22}\right) ; \\
& \tau_{3}=\left[-\frac{1}{2}(\cos 2 \phi+\sqrt{3} \sin 2 \phi) \sigma_{12}\right. \\
&\left.+\frac{1}{4}(\sin 2 \phi-\sqrt{3} \cos 2 \phi)\left(\sigma_{22}-\sigma_{11}\right)\right]
\end{aligned}
$$

with

$$
\begin{gathered}
\sigma_{11}=\sigma_{\mathrm{r}} \cos ^{2} \theta+\sigma_{\theta} \sin ^{2} \theta-\sigma_{\mathrm{r} \theta} \sin 2 \theta, \\
\sigma_{12}=\left(\sigma_{\mathrm{r}}-\sigma_{\theta}\right) \frac{\sin 2 \theta}{2}+\sigma_{\mathrm{r} \theta} \cos 2 \theta, \\
\sigma_{22}=\sigma_{\mathrm{r}} \sin ^{2} \theta+\sigma_{\mathrm{o}} \cos ^{2} \theta+\sigma_{\mathrm{r} \theta} \sin 2 \theta .
\end{gathered}
$$

In the test reported here above (Fig. 5) it can be noticed that far from the cavity only Systems 1 and 3 are active; since the lowest Schmid factor of these systems is equal to 0.33 , this value will now be considered as critical for the activation of a system. Thus, the different zones where this criterion is reached can be determined (Fig. 7); Systems 1 and 3 are activated everywhere except in the pole regions. The perturbation of the applied stress field by the cavity induces areas where System 2 is activated. The different activation zones are in good agreement with the observed slip traces (Fig. 5). The variation of the resolved shear stress of System 2 around the hole (Fig. 7) shows that its sign changes, systematically inducing a centrifugal glide consistent with the growth of the cavity. 


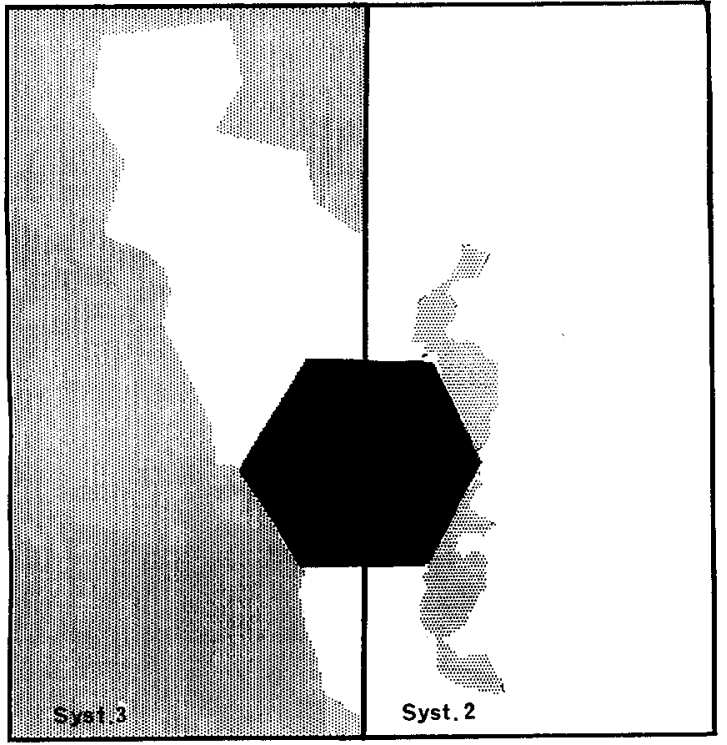

Fig. 8. Results of a finite element calculation for an elastic-plastic material with kinematical hardening. Left part: representation of the area (shaded) where the criterion is reached on System 3 for a semi-hexagonal cavity. The area of activation of System 1 is obtained by symmetry with respect to the horizontal axis. Right part: representation of the areas where the criterion is reached on System 2 for a semi-hexagonal cavity.

Another solution of this problem consists of taking a finite element approach with an elastic-plastic and kinematical hardening behavior as a constitutive relation. The result of the calculation is presented in Fig. 8 for the same activation criterion as above. The three prismatic slip systems are also activated two by two around the cavity and the stress concentration factors on each of the systems are equal, which means that there is the same quantity of gliding on the three slip systems.

The question about the activation by circular permutation of the three prismatic slip systems finds a satisfactory qualitative answer in this simplified approach.

\section{Morphological stability}

The stability of the hexagonal morphology can be discussed by analogy between the behavior of each of the six angles of the cavity and the observations made by Neumann [8] and Pelloux [9] of the crack propagation without blunting occurring in a single crystal in double glide under tension. These authors consider that the crack propagates by alternate
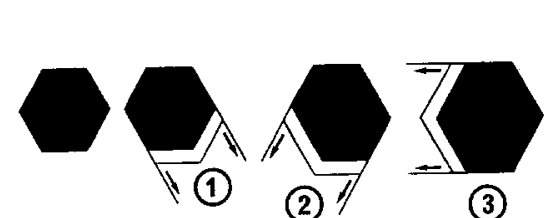

(3)

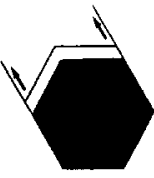

(4)
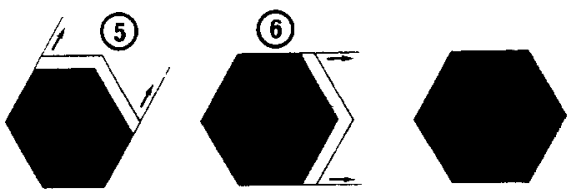

Fig. 10. Decomposition of a kinematical model based on activation of the three prismatic systems two by two around the cavity with the same amount of gliding.

activation of the two slip systems concentrated at the crack tip (Fig. 9). This model can be adopted to simulate the growth behavior in each of the six angles by activation of two prismatic slip systems with the same quantity of gliding; this assumption is in agreement with the results of the finite element calculation. A simulation is presented in Fig. 10 by means of a decomposition in six steps, which correspond to one increment of growth; this kind of representation justifies the homothetic growth of the cavity. Of course, it must be assumed that a certain amount of hardening on an active slip system induces its disactivation and the activation of another one. That hardening may be due to a dislocation pile-up, induced by the stress gradient $\left(1 / r^{2}\right)$ around the cavity, which produces a strong decrease in dislocation velocity. If the traces of the slip bands are kept around the cavity and their length is taken equal to the present size of the cavity, the previous simulation conducts to the apparition of triangular patterns that compares favorably to the experimental ones (Fig. 5). Moreover, this kinematical model applied to an initially circular cavity transforms its shape to a hexagon during one increment of growth.

\section{Growth kinetics}

In the kinematical model, cavity growth occurs by the creation of steps due to the plastic slip resulting from the nucleation and propagation of dislocations in the vicinity of the hexagon corners. The length $(l)$ of the resulting slip bands can be taken proportionally to the cavity size $(R)$ :

$$
1=\alpha R, \quad \text { with } \alpha \approx 1 .
$$

The longer the band the larger is the number of dislocations in the corresponding pile-up and the

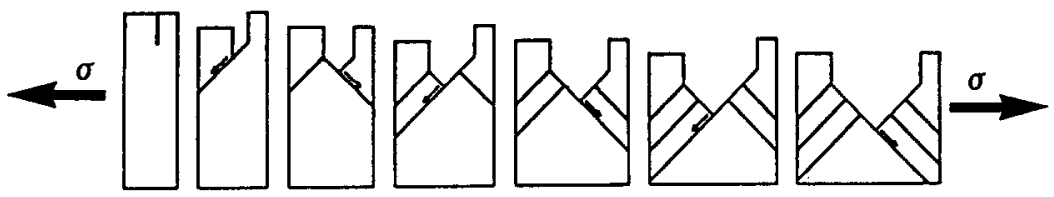

Fig. 9. Model proposed for crack opening under tension by alternate activation of two slip systems [8]. 
larger is the step. If $e$ is the step size, in first approximation:

$$
e=\beta 1,
$$

with $\beta$ a proportionality factor.

For geometrical reasons only, the homothetic growth of the cavity imposes that the shear magnitude in each slip band is $\gamma=1$ near the cavity.

Under the assumptions that the macroscopic Norton-Hoff (2) law applies at the shear band scale:

$$
\tau=\tau_{0}\left(\frac{\dot{\gamma}}{\dot{\gamma}_{0}}\right)^{m}
$$

and that the shear stress $\dot{\gamma}_{\infty}$ far from the cavity is induced by a shear stress $\tau_{\infty}$, the shear strain at a corner of the cavity under the local shear stress $k \tau_{\propto}$ obeys:

$$
\dot{\gamma}=\dot{\gamma}_{\infty} \mathbf{k}^{1 / m}
$$

where $\mathrm{k}$ is a stress concentration factor. The time for the formation of one step, i.e. for reaching $\gamma=1$, near the cavity is:

$$
\theta=\frac{\gamma}{\dot{\gamma}}=\frac{1}{\dot{\gamma}_{\infty} \mathbf{k}^{1 / m}} .
$$

One increment of growth for the cavity needs six steps (Fig. 10) and a time $\Delta t=6 \theta$; during that time the cavity grows by $\Delta R=2 e$. Then the cavity growth rate is:

$$
\frac{\Delta R}{\Delta t}=\frac{2 e}{6 \theta}=\frac{1}{3} e \dot{\gamma}_{x} \mathrm{k}^{1 / m},
$$

and after combination with equations (14) and (15):

$$
\frac{1 \Delta R}{R \Delta t}=\frac{1}{3} \alpha \beta \dot{\gamma}_{\infty} \mathrm{k}^{1 / m}
$$

In the considered geometry:

$$
\dot{\boldsymbol{\epsilon}}_{x}=\frac{\sqrt{3}}{4} \dot{\gamma}_{x},
$$

equation (20) is transformed into

$$
\frac{\dot{R}}{R}=\frac{4 \alpha \beta}{3 \sqrt{3}} \mathrm{k}^{1 / m} \dot{\epsilon}_{\infty}
$$

The micrograph (Fig. 5) reveals that $1 \approx 5 \mu \mathrm{m}$ and $e \approx 0.5 \mu \mathrm{m}$. So, it is consistent to propose for $\alpha=1$ and $\beta=0.1$, and consequently:

$$
\frac{\dot{R}}{R}=8 \times 10^{-2} \mathrm{k}^{1 / m} \dot{\boldsymbol{\epsilon}}_{\alpha_{*}}
$$

Thus, for the present model:

$$
\zeta=8 \times 10^{-2} \mathrm{k}^{1 / m}
$$

Results from the literature [10] allow one to estimate $m$ to be in the interval:

$$
0.02 \leqslant m \leqslant 0.03 \text {. }
$$

These values inserted in equation (23) give a very high growth rate if the concentration factor $\mathrm{k}$ is greater than 1. This case should correspond to the stress state close to the corners of the hexagon. This value is not explicitly determined by an analytical solution, but a finite element approach, with a crystalline finite element model, could afford the determination of the concentration factor. However, it seems to be reasonable to take one in the order of 1.2 or 1.3 . It follows:

$$
\begin{aligned}
& \frac{\dot{R}}{R} \simeq 100 \dot{\epsilon}_{\infty}, \quad \text { for } \mathrm{k}=1.2 \\
& \frac{\dot{R}}{R} \approx 3000 \dot{\epsilon}_{\infty}, \quad \text { for } \mathrm{k}=1.3 .
\end{aligned}
$$

Then for the cases where the stress sensitivity factor $m$ is weak but nonequal to 0 this kind of model can afford to explain the very fast cavity growth rate $(\zeta$ coefficient) and the stability of the hexagonal shape, observed experimentally.

\section{DISCUSSION}

The model presented is based on a description of cavity growth and accounts for crystallographic slip localized in narrow bands, which develop at the corners of the hexagonal cavity. It explicitly accounts for the effect of local stress state near these peculiar points and introduces the material constitutive relation in the growth rate equation. The $\zeta$ coefficient, which characterizes the cavity growth rate (23) is directly proportional to the local stress concentration factor and depends on the stress sensitivity factor $m$.

In the classical models based on continuum mechanics (e.g. McClintock [6] or Rice and Tracey [5]) the driving force for cavity growth is the global state of stress triaxiality and not the local stress; moreover, in these models there is no real free surface formation since there is not any discontinuity during the cavity growth, two neighboring points remain neighbors. The model presented gives a more physical description of the growth of cavities.

To investigate the difference between these two types of models, two extreme cases are considered: a Newtonian material $(m=1)$ and a perfectly plastic material $(m=0)$.

A Newtonian material shows a weak stress sensitivity and a growth rate coefficient $\zeta$ proportional to the stress concentration factor $\mathrm{k}$, near unity. In that case the crystallographic model gives a growth rate one order of magnitude lower than the continuum mechanics models $\zeta \approx 1$ as shown in Fig. 6.

A perfectly plastic material shows an infinite stress sensitivity or in other words no strain hardening. The crystallographic model predicts an infinite growth rate; this value represents the unstable behavior of a crystal without strain hardening: plastic glide concentrates in a slip line impeding the activation of other systems (except if geometrical hardening is active). Therefore, cavity nucleation is impossible and 
rupture occurs by concentrated glide. Nevertheless continuum mechanics models predict a $\zeta$ value still close to unity $(\zeta \approx 1.2)$.

Thus, the crystallographic approach of cavity growth shows a much stronger sensitivity to the material behavior than the continuum mechanics ones, due to the introduction of the local stress state and of the crystallographic glide. This type of crystallographic approach imposes the knowledge of the constitutive relation of the material and its application to the local scale on each slip system. This is a very important assumption, but for zirconium deformed at room temperature, a "Norton-Hoff". type constitutive relation seems to give a good result. This good result is certainly due to the fact that at room temperature there is only one type of slip system activated, the three prismatic systems.

\section{CONCLUSION}

Damage mechanisms in $\beta$-treated zirconium are linked to the principal deformation mechanism, prismatic slip. The main damage mechanism is the early nucleation and fast growth of tubular cavities. The slip localization along lath boundaries forces these cavities to form alignments parallel to these boundaries; rupture occurs by crack propagation along the dotted lines formed by the cavities. The axis of the cavities is parallel to the $\langle c\rangle$ axis of the crystal and, for crystallographic orientations of the lath inducing double glide, these cavities have a hexagonal cross-section. A crystallographic model accounts for the creation of free surface by localization of deformation and explains the hexagonal shape and the very fast cavity growth rate.

Acknowledgement-The authors wish to thank the CEA/CEREM for supplying both materials and financial support.

\section{REFERENCES}

1. J. Crépin, T. Bretheau and D. Caldemaison, Acta metall. mater. 43, 3709 (1995).

2. L. Allais, M. Bornert, T. Bretheau and D. Caldemaison Acta metall. mater. 42, 3865 (1994).

3. I. Aitchinson and B. Cox, Corrosion-NACE 28(3), 83 (1972).

4. J. Spurrier and J. C. Scully, Corrosion-NACE 28(12), 453 (1972).

5. J. R. Rice and D. M. Tracey, J. Mech. Phys. Solids 17, 201 (1969).

6. F. A. McClintock, J. appl. Mech. ASME 35, 363 (1968).

7. S. Timoshenko, Theory of Elasticity. McGraw-Hill, New York (1951).

8. P. Neumann, Acta metall. 22, 1167 (1974).

9. R. M. Pelloux, Proc. Air Force Conf. on Fatigue and Fracture of Aircraft. Structures and Materials, Miami Beach, p. 409 (1969).

10. P. Lemoine, T. Forgeron, F. Le Naour, B. Marini and M. Mottot, J. Études Zirconium SF2M, 75 (1990). 